\title{
A simple discriminant function for hepatic disease
}

\author{
MAX HAMILTON \\ From the Department of Psychiatry, The University of Leeds, 15 Hyde Terrace, Leeds, LS2 9LT
}

SUMmARY Tests of hepatic function do not correlate highly and this, together with their skew distribution, makes the information provided by them difficult to interpret. By means of an appro- $-\bar{\rho}$ priate transformation it is possible to normalise their distributions so that the information from them can be combined in the form of a discriminant function which is not only easy to interpret but $O$ gives an index of severity of disease which is more reliable than any one single test. In this study if nine tests were examined, and after normalisation it was found that only four tests were required to + present almost all the information available in the nine. A formula is given for calculating the index 9 in T-score form, which is particularly easy to interpret.

A discriminant function of the type described retains the maximum information with the minimum number of biochemical tests. It therefore contributes to the solution of a problem which is causing increasing concern-the exponential increase in the number of investigations which the hospital $\underset{\forall}{\mathcal{V}}$ services will soon find it impossible to bear.

Tests of hepatic function are used for determining the presence of damage to the liver, qualitatively in the differentiation between normal and disease and quantitatively for the measurement of improvement or deterioration. These purposes are by no means the same. For example, the presence of disease might give rise to the appearance of an abnormal metabolite and this would be of diagnostic value; but if the metabolite were not related quantitatively to the severity of disease it could not be used for the measurement of improvement or deterioration. In statistical terms, a test which is used to differentiate normal from disease should show a large significant difference between means of the two conditions, that is, have a small overlap of the distributions, but a test which is selected to measure deterioration or improvement should correlate highly with changes in function. Most tests of hepatic function measure the changes in concentration of metabolites which are normally present. They therefore measure severity of disturbance of function and can be used to detect disease only when the amount of change is such that the probability of such results being obtained from normal livers is sufficiently low.

It is well known from clinical experience that when a battery of tests of hepatic function is used the amount of change shown by them will differ considerably, depending upon the underlying pathology

Received for publication 13 October 1976 and disturbance of function. The lack of correspondence between test results can be easily demon- $\frac{\circ}{\mathbb{D}}$

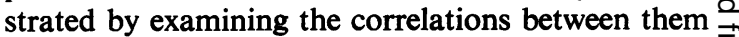
(Miloszewski et al., 1970). Table 1 is a set of $\frac{\vec{\rho}}{3}$ correlations between nine tests obtained from a group of 73 patients suffering from a variety of liver diseases. It can be seen that most of the correlations are quite low and many do not differ significantly용 from a zero correlation. (As serum albumin moves $\overline{-}$ in the reverse direction to the others in hepatic disease, its negative correlations are a special case.) $ᄋ$

For test scores to be regarded as adequate $₹$ measures of changes in function it is important that $ᄋ$ the relationship between the two should be not only $>$ simple but also easy to interpret. This is by no means common. The frequency distribution of most bio- N chemical tests on normal subjects is asymmetrical, the mode being close to the low scores with a long $N$ tail towards the higher scores. This means that high scores are more common than low ones and therefore 0 since high scores imply abnormal functioning, such 0 tests might lead to a biased interpretation towards $\frac{\widetilde{\Phi}}{\mathbb{}}$ abnormality. Although this has been well known to $\stackrel{\oplus}{+}$ clinical pathologists for many years, the implications 0 for clinical work have not been adequately utilised, $\stackrel{\vec{P}}{\circ}$ and reports are still given in terms of actual readings $\stackrel{\square}{\square}$ without indicating the distribution in normal sub- $\mathbb{D}$ jects. Statistical tests of significance can also be misleading in such circumstances. In order to overcome this difficulty it is necessary to transform the 
Table 1 Correlation matrix of nine tests of hepatic function based on 73 cases of hepatic disease

\begin{tabular}{|c|c|c|c|c|c|c|c|c|c|c|}
\hline 1 & Total bilirubin & 1.00 & & & & & & & & \\
\hline 2 & Prothrombin time & 0.29 & 1.00 & & & & & & & \\
\hline 3 & Alanine amino-transferase & 0.55 & $0 \cdot 14$ & 1.00 & & & & & & \\
\hline 4 & Albumin & $-0 \cdot 36$ & -0.27 & -0.12 & 1.00 & & & & & \\
\hline 5 & Globulin & $0 \cdot 15<$ & $0 \cdot 18$ & $0 \cdot 13$ & -0.26 & 1.00 & & & & \\
\hline 6 & Zinc turbidity & -0.26 & $0 \cdot 16$ & -0.07 & -0.20 & 0.61 & 1.00 & & & \\
\hline 7 & Thymol turbidity & -0.00 & 0.08 & 0.14 & $-0 \cdot 23$ & 0.53 & 0.73 & 1.00 & & \\
\hline 8 & Alkaline phosphatase & 0.47 & 0.23 & 0.34 & $-0 \cdot 27$ & 0.22 & $-0 \cdot 10$ & 0.06 & 1.00 & \\
\hline 9 & 5-Nucleotidase & 0.41 & 0.33 & 0.32 & -0.30 & $0 \cdot 25$ & 0.02 & $0 \cdot 14$ & 0.78 & 1.00 \\
\hline
\end{tabular}

Before calculating the correlations, the data were transformed as shown in Table 2

A correlation of $\mathbf{0 . 2 3}$ or less does not differ significantly from zero at the $\mathbf{0 . 0 5}$ level

original data in such a way as to produce a distribution which is more or less symmetrical. In most cases it is sufficient to use a logarithmic function of the scores. This was necessary in seven of the nine tests used in this paper, the two exceptions being serum albumin and serum globulin.

The effect of logarithmic transformation of the scores is shown (Fig. 1). When a frequency distribution of Gaussian form is plotted in the usual way it produces the familiar bell-shaped curve. When the distribution is plotted as a cumulative distribution, that is, the height of the curve for any one score represents the total frequency of cases up to that score, the curve has a stretched-out $\mathbf{S}$ shape. When this curve is plotted on probability paper it becomes a straight line (Hamilton, 1974). In Fig. 1 the curve is drawn for alanine amino-transferase, and it is clear that then the data are converted into their logarithmic equivalents; the curve of the cumulative frequency distribution is effectively straightened out.

Clinical experience has shown that, both for establishing the presence of disease and for assessing progress, reliance cannot be placed on one single

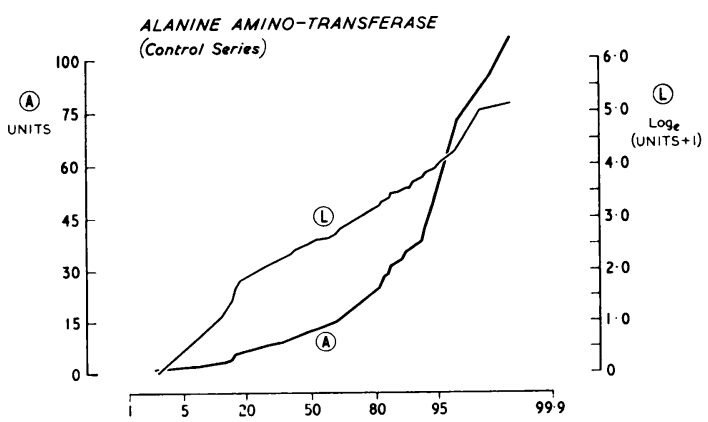

Fig. 1 Cumulative frequency distribution plotted on probability paper. The log transform has effectively straightened out the curve, showing that the asymmetrical distribution has been normalised. test and therefore the physician commonly uses several. In some unspecified way he combines the information from several tests and comes to a decision. It would be highly desirable if some explicit method were available for combining the test scores so as to give the maximum information. This would give an index which would be closely related to the level of hepatic function, that is, had high validity, and which differentiated efficiently between normal and diseased livers, that is, had high discriminative power.

It has been shown by Lubin (1950) that the best combination of a set of variables is a simple linear function, ie, a weighted sum of the test scores, provided that the variables are multivariate normally distributed and that their dispersions are homogeneous. This applies equally when a dependent variable is being estimated by means of multiple regression, or when distinct groups are being differentiated by means of a discriminant function. When there are only two groups, ie, the criterion is dichotomous, the two mathematical techniques give identical results. This applies in the present case where the groups consist of healthy and diseased livers.

The procedure for finding the best combination of test scores is as follows: the results from the nine tests shown in Table 2 were available for 73 patients suffering from liver disease and for 97 controls (patients in hospital not suffering from any condition affecting hepatic function). A tenth variable was created by assigning to each control case a score of 1 and to each patient a score of 2 . Such a variable is known as a dichotomous criterion variable because each person's score assigns him to one of two groups. The intercorrelations were then calculated. The size of a correlation between a test and the criterion, as shown in Table 2, is a measure of the efficiency with which the test distinguishes between the two groups. It can be seen that this varies considerably from the highest figure of 0.53 for alkaline phosphatase to the lowest of 0.14 for prothrombin time. The best combination (weighted sums) of the tests is found by 
calculating the multiple regression of the nine tests on the criterion. The nine weights so obtained are used to multiply the corresponding scores of each individual on the nine tests and the products are summed. This gives an index number which, when correlated with the criterion variable, gives a cor-

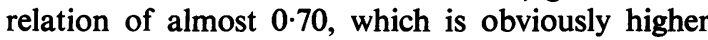
than the best individual test. The weights are given, in the last column of Table 2, in standardised form which indicates clearly the relative importance of the tests in contributing to the total. Some of the weights are very small, which indicates that those tests are making a negligible contribution to the index number and could therefore be omitted without making any real difference.

The deletion of 'non-contributory' tests is done systematically with a computer program 'Multiple correlation by stepwise accretion and deletion' from the PLUS System (Hamilton, 1965). This program deletes the test with the lowest weight and recalculates the multiple regression and correlation. It then removes the test with the lowest weight and so on. It also works the reverse way by starting with the best single test, that is, the one with the highest correlation with the criterion, and then successively adds tests, choosing that test among the remaining ones which makes the biggest increase in the multiple correlation. Both ways show that four tests give a multiple correlation which does not differ significantly from that obtained with all nine.

This is demonstrated by the analysis of variance in Table 3. Certain statistical difficulties have to be overcome. The usual method of calculating discriminant functions assumes that the dispersion matrices of the two groups do not differ significantly, but it has been shown that they do indeed differ (Winkel and Juhl, 1971), the variances of the group of liver disease cases being greater than that of the controls. The method used, therefore, was to com-

Table 2 Multiple correlation and regression:proportionate weights

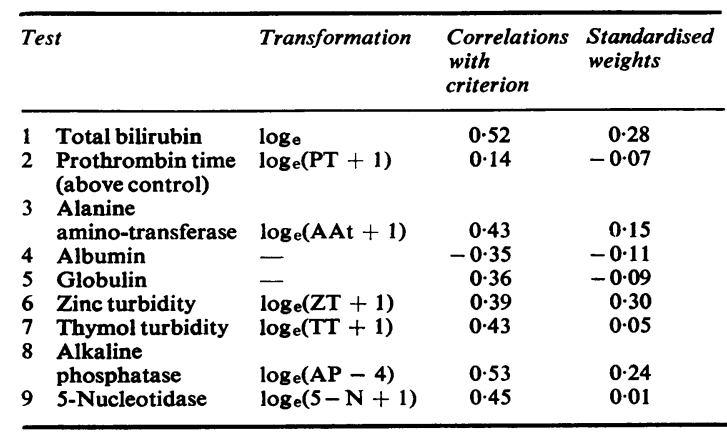

Multiple R $=0.696$ bine the data of the two groups and to calculate multiple correlation and regression weights. For the linear function, the weights so obtained were applied to the dispersion matrix of the control group alone in order to calculate the weights of the regression formula. Strictly speaking, a non-linear regression should have been used because of the non-homogeneity of variances. This was done, using the linear function as one variable and the squares and crossproducts of the four constituent tests as the others. This gave a multiple correlation of 0.712 , which is not significantly better than the linear function. The latter is therefore adequate for the purpose.

Four tests only are as effective as the full nine, and these are total bilirubin, alanine amino-transferase, zinc turbidity, and alkaline phosphatase. Their standardised weights, which show the relative importance of their contribution to the index, are respectively $0.27,0.16,0.29$, and 0.26 . They give a multiple correlation of 0.685 , which is not significantly less than 0.696 obtained with all nine tests.

For the convenience of the user, the formula for $\vec{\theta}$ the index (discriminant function) has been put into. $\mathcal{}$ 'T-score form'. This means that the discriminant score for the control population has a mean of 50 and a standard deviation of 10 . Provided that the discriminant score is normally distributed (and statistical tests show that this is true for both the control series and the series of patients) then it is known that $2.5 \%$ of the control population can be expected to have a score of 70 points or over, $0.6 \%$ will have a score of 75 or over, and $0.1 \%$ will have a score of 80 or over. Thus a score of between 70 and 80 suggests that there may be hepatic impairment, and a score of 80 or over indicates that disturbance of function is almost certainly present. The nature of the tests indicates that the index can also be used to assess changes in function. The formula for the index is as follows:

Discriminant function score $=7 \cdot 65 X_{1}+3.36 X_{2}+$ $8.33 X_{3}+8 \cdot 19 X_{4}$ or (in whole numbers) $=1 / 6$

Table 3 Analysis of variance for multiple regression

\begin{tabular}{|c|c|c|c|c|c|}
\hline Source & $d f$ & $\begin{array}{l}\text { Sum of } \\
\text { squares }\end{array}$ & $\begin{array}{l}\text { Mean } \\
\text { square }\end{array}$ & $F$ & $\mathbf{P}$ \\
\hline $\begin{array}{l}\text { Regression on } 3 \text { best } \\
\text { variables }\end{array}$ & 3 & 0.453 & $0 \cdot 151$ & $46 \cdot 85$ & 0.0005 \\
\hline $\begin{array}{l}\text { Regression on fourth } \\
\text { variable }\end{array}$ & 1 & 0.016 & 0.016 & 5.06 & 0.05 \\
\hline $\begin{array}{l}\text { Regression on last five } \\
\text { variables }\end{array}$ & 5 & 0.015 & 0.003 & 0.94 & NS \\
\hline $\begin{array}{l}\text { Deviation from } \\
\text { regression } \\
\text { Total }\end{array}$ & $\begin{array}{l}160 \\
169\end{array}$ & $\begin{array}{l}0.516 \\
1.000\end{array}$ & 0.003 & & \\
\hline
\end{tabular}

The table shows that, added to the first three, the fourth test just makes a significant contribution $(P<0.05)$. The last five tests, either singly or together, do not make a significant contribution. 
$\left(46 X_{1}+20 X_{2}+50 X_{3}+49 X_{4}\right)$, where $X=\log _{\mathrm{e}}$ (total bilirubin mmol/l), $X_{2}=\log _{e}$ (alanine aminotransferase units +1$), X_{3}=\log _{e}$ (zinc turbidity units +1 ), and $X_{4}=\log _{\mathrm{e}}$ (alkaline phosphatase units - 4). If total bilirubin is measured in $\mathrm{mg} / 100$ $\mathrm{ml}$, add a constant of 21.71 ; for the integer form add 131 inside the brackets.

If an index is to be of practical value it should be easy to calculate and simple to understand. The diagram at the end of this paper (Fig. 3) provides a simple way of calculating the index. The index numbers corresponding to the four test results are read off and added together.

One way of considering the power of a clinical test is to examine the overlap of the distribution for patients and for the controls. The overlap will depend to some extent on the mode of selection of patients. As the present series is a consecutive one of admissions, it can be regarded as representing the general run of patients. The data are somewhat surprising. From this point of view zinc turbidity is of least value as the lowest and highest scores are identical for patients and controls. For total bilirubin, prothrombin time, thymol turbidity, alkaline phosphatase, and 5-nucleotidase the lowest scores are the same in both groups. For these tests the number of liver disease patients whose scores on the tests exceed the highest score of the control group is, respectively, 19, 2, 8, 22, and 16 out of 73 . For globulin, one patient has a lower score than the controls and four are higher; and for alanine amino-transferase, five are below and 12 higher.
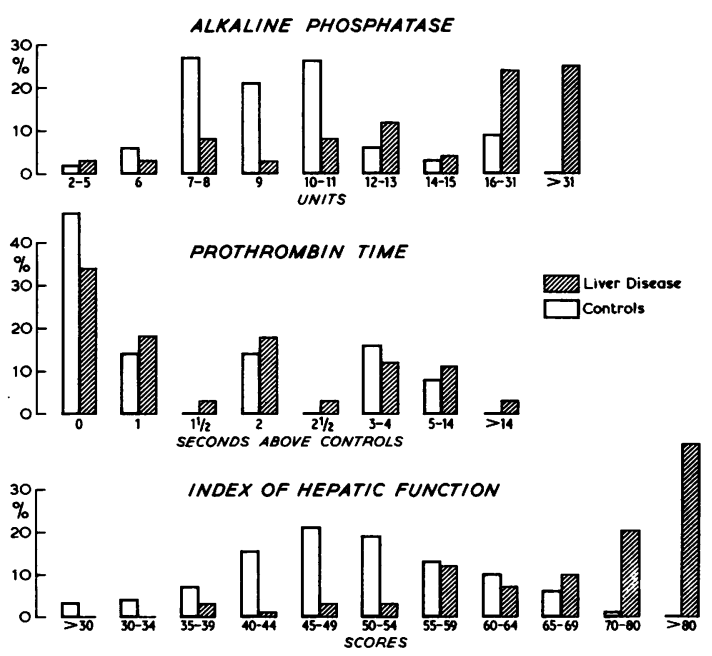

Fig. 2 Bar diagram showing the percentage frequency distribution of scores on two tests and the index of hepatic function
Serum albumin moves in the reverse direction from other tests, and for this test only one patient is below the controls and four are higher. The results obtained with the liver function index is a considerable improvement on these figures: 42 of the 73 patients have scores higher than the highest in the control series and 13 of the control series have scores below the lowest of the patients' scores. Figure 2 illustrates these figures for two tests and the index. It shows the frequencies, expressed as percentages for scores grouped by intervals of about half a standard deviation (shown most clearly for the index) except at the right-hand tail. These results depend on the extreme scores and obviously could vary considerably from one sample to another. It would be better to fit distribution curves and calculate the percentage of overlap or of correct classification for these curves (Levy, 1967). This gives $71 \%$ and $70 \%$ correct classification for alkaline phosphatase and total bilirubin, respectively, down to $42 \%$ for serum albumin. In comparison, the linear discriminant index gives $83 \%$. Much the same information is obtained from examining the correlations of the tests with the (dichotomous) criterion, as in Table 2.

In ordinary clinical practice it is not always possible to have all biochemical tests done when required. Thus, if four tests have been ordered, only three may have been performed. It is still possible to calculate the index even though it is a little less reliable with three variables than with the full four. Table 4 gives the weights and constants for calculating the index for any combination of three variables. Among the four versions, the third one, which omits alanine amino-transferase, is the most efficient, but the second one, which omits the test of zinc turbidity, is probably the most convenient. The laboratory results are transformed in the usual way, multiplied by the weights and the constant added. Many hospitals now have available a modern programmable calculator for which a simple program can be written to produce the index from the original laboratory results. Even a cheap calculator with a single memory and log key will complete the calculation in less than 30 seconds.

Table 4 3-Variable index of hepatic function

\begin{tabular}{|c|c|c|c|c|}
\hline \multirow{6}{*}{$\begin{array}{l}\text { Total bilirubin ( } \mu \text { mol/1) } \\
\text { Alanine amino-transferase (units) } \\
\text { Zinc turbidity (units) } \\
\text { Alkaline phosphatase } \\
\text { Constant }\end{array}$} & \multicolumn{4}{|c|}{ Weights } \\
\hline & $7 \cdot 40$ & 6.41 & 7.90 & - \\
\hline & $5 \cdot 21$ & $6 \cdot 24$ & - & $6 \cdot 32$ \\
\hline & $10 \cdot 48$ & - & $11 \cdot 34$ & $8 \cdot 73$ \\
\hline & - & $9 \cdot 41$ & $7 \cdot 82$ & $7 \cdot 97$ \\
\hline & 6.06 & 5.69 & $4 \cdot 11$ & $7 \cdot 23$ \\
\hline
\end{tabular}

Logarithmic transformation of variables as before

To calculate the index of hepatic function, using only three tests, transform the test results as shown in Table 2, multiply by the appropriate weight, and add, together with the constant. 


\section{NOMOGRAM FOR INDEX OF HEPATIC FUNCTION}

Total Bilirubin

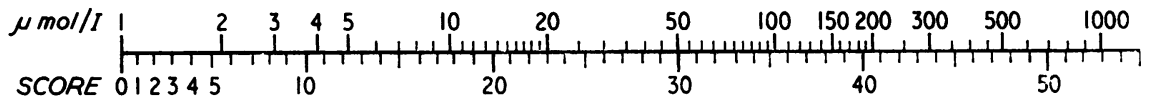

Alonine omino-tronsferose

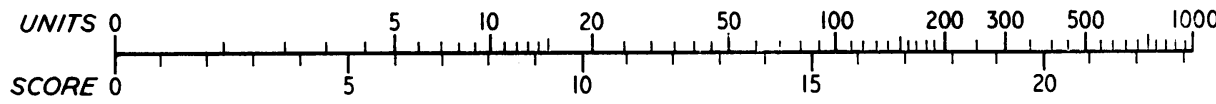

Zinc Turbidity

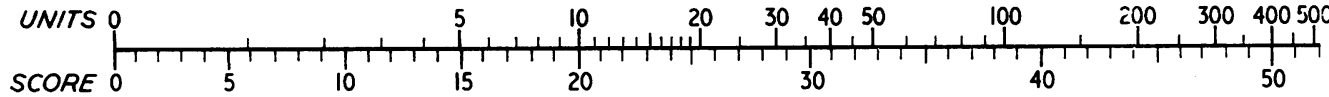

Alkoline Phosphatose

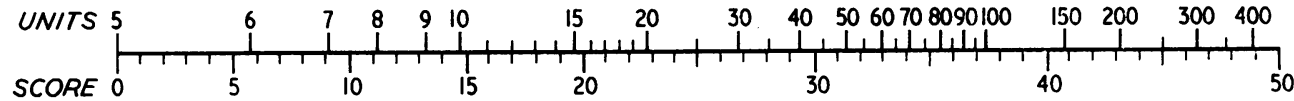

Fig. 3 Nomogram for calculating the index of hepatic function. The scores corresponding to the four test results are read and summed. (Large-scale copies of the nomogram may be obtained from the author.)

\section{Discussion}

Biochemical tests are used for the measurement of hepatic function but those currently available are not entirely satisfactory for this purpose. This is shown both by the continued search for better tests and also by the fact that, even when a diagnosis has been made, for the assessment of progress most clinicians are not satisfied with the results obtained from one test only but use several. It is not satisfactory to use a large battery of tests in ordinary clinical practice, partly because this adds to the burden on the laboratories and to the cost, and also because of the difficulty in collating the information. The low correlations between tests indicate that the greater the number of tests used, the more likely is it that considerable discrepancies between test results will be found, making interpretation difficult. The discriminant function provides a solution to these difficulties. It indicates how many tests and which ones are required to provide the desired information, combines them so as to provide the maximum differentiation between 'normal' controls and patients with liver disease, and also provides quantitative information for monitoring the changes produced by disease and during recovery.

A discriminant score gives the best single index for the assessment of function but it is less useful for the purposes of deciding if disease is present. This requires a consideration of the overlap of frequency distribution of scores from normals and patients which depends not only on the distance between the means of these two groups but also on their relative frequencies. Such frequencies should ideally be determined for each hospital and indeed for each physician. The enthusiast who orders tests too frequently actually diminishes the probabilities which enable him to make a diagnosis of the presence of disease, even though the percentages of misclassifications are independent of relative frequencies.

There are other difficulties. Hepatic function may remain within normal limits despite the presence of obvious pathological changes; in such cases discriminant scores will not aid diagnosis. For this reason biochemical tests do not supplant the use of $\mathrm{O}$ liver biopsy, but this must not be undertaken lightly, $N$ and in any case it cannot be repeated frequently for monitoring purposes.

There is nothing particularly new in this investigation. Zieve and Hill (1955) constructed a discriminant function for hepatic disease 20 years ago. Why did their work not achieve wide acceptance? A partial answer to this question is that it is often the misfortune of pioneers to be premature. Perhaps the present generation of clinicians may be more sympathetic to this approach. Zieve and Hill used their discriminant function for diagnosis rather than for monitoring. They did not correct for skew dis- 
tributions (although they recognised their presence), which would give rise to many false positives. The tests used in this study are not the same as those used by Zieve and Hill. Although they also found that four tests were sufficient, the ones they selected were bromsulfalein, zinc turbidity, hippuric acid, and urine coproporphyrin. Clinical experience has led to the abandonment of old tests and their replacement by new ones. Even those used in this study are beginning to have an old-fashioned look about them. It is, however, of interest that the test which emerges with the highest weighting in the function is the zinc turbidity, a test which unfortunately is falling into disuse in recent years because it cannot be mechanised. Contrary to what might appear at first sight, the continued introduction of new and better tests can enhance the value of a discriminant function in the form of $\mathrm{T}$-scores. As further tests come into use, they can be combined with the discriminant function, either as an addition to, or as a replacement for, the tests already included. The function becomes more efficient but its 'appearance', ie, the mean and standard deviation of controls, remains the same so that the clinician does not have to learn to interpret a new test with its own particular mean, standard deviation, and frequency distribution.
I thank Professor M. Losowsky who not only provided the data but also gave me help and guidance. I also thank Mr H. G. Lumby, medical illustrator, for the figures, and Mrs J. M. Hanning for her care and attention in preparing the manuscript.

\section{References}

Hamilton, M. (1965). Computer programmes for the medical man: a solution. British Medical Journal, 3, 1048-1050.

Hamilton, M. (1974) Lectures on the Methodology of Clinical Research, 2nd edition. Churchill Livingstone, London \& Edinburgh.

Levy, P. (1967). Substantive significance of significant differences between two groups. Psychological Bulletin, 67, 37-40.

Lubin, A. (1950). Linear and non-linear discriminating functions. British Journal of Psychology, 3, 90-104.

Miloszewski, K., Walker, B. E., Hamilton, M., and Losowsky, M. S. (1970). A new look at biochemical tests of liver function. European Journal of Clinical and Biochemical Research, 15, 878-881.

Winkel, P. and Juhl, E. (1971). Assumptions in linear discriminant analysis (Letter). Lancet, 2, 435-436.

Zieve, L. and Hill, E. (1955). An evaluation of factors influencing the discriminative effectiveness of a group of liver function tests: III. Relative effectiveness of hepatic tests in cirrhosis. Gastroenterology, 28, 785-802. 